\title{
Component separation technique for closure of burst abdomen
}

\author{
Ashraf Goda, MD; Mohamed Nasr, MD; Osama H Gharib, MD; \\ Mostafa B Mohamed, MD
}

General Surgery Department, Faculty of Medicine, Zagazig University.

Background: Burst abdomen is a continuing problem for the general surgeon as the incidence of such complication may reach $3 \%$ with a mortality rate exceeding $25 \%$.

Methods: Component separation of all layers of anterior abdominal wall would give an extra length on each side towards medial advancement. 20 patients, 12 men and 8 women aged 35-65 (mean, 48.6) years, with burst abdomen after major gastrointestinal and hepatobiliary surgery whose abdomen could not be closed primarily were managed using this technique. Long term follow up of patients was done for development of complications.

Results: One patient died on the fourth postoperative day from pulmonary embolism (mortality 5\%), and 2 patients developed subincisional abscesses (10.5\%). Skin necrosis occurred in 1 patient (5\%). No single case of reburst occurred. Only one patient showed few symptoms of abdominal compartment syndrome. The mean hospital stay was 18.5 days. Long term follow up showed only one case of incisional hernia (5.5\%) in a patient with biliary fistula.

Conclusion: The component separation technique is a suitable, straightforward and tension free method, with a comparable mortality and morbidity to other conventional methods for closure of burst abdomen.

Key words: Component separation, burst abdomen, abdominal closure.

\section{Introduction:}

Burst abdomen is a continuing problem for the general surgeon, as the incidence may reach $3 \%$ of major laparotomy wounds ${ }^{1}$ with mortality rate ranging from $24 \%{ }^{2}$ to more than $44 \% .^{3}$ There was no difference in mortality between patients with evisceration and those with dehiscence only. ${ }^{4}$ The closure under tension is doomed to failure so that if we are unable to reapproximate the fascia without tension, then we will usually have to place a fascial substitute. Many different methods have been used to close the dehiscence, ranging from simply closing the skin over the defect and leaving the fascia and peritoneum wide open to doing relaxing incisions of both fascia and skin well away from the wound, closing the primary wound and skin grafting the resulting defect. More recently, the vacuum pack technique, ${ }^{5}$ with mesh of some type, has been used, including Proline, Marlex, PTFE and Vicryl, or more complex closures using pedicled or rotation flaps are being used.6,7 None of these methods proved to be ideal or without a significant hazard, and certain disadvantages inherent in each technique require departure from the ideal: primary tension-free parietal closure without use of prosthetic material. ${ }^{8}$

\section{Patients and methods:}

After obtaining Institutional Review Board approval, we studied all patients undergoing component separation technique for closure of burst abdomen from January 2009 to February 2011 at the Emergency Unit of Zagazig University Hospital. All patients included developed burst abdomen between the $7^{\text {th }}$ and $15^{\text {th }}$ day after major gastrointestinal operations done through a longitudinal abdominal incision. All were taken to surgery once their bursts were discovered. Analysis for patient demographics including sex, age, original surgery and hospital stay was done 
Table (1). One patient had liver cirrhosis and developed postoperative ascites after the first operation before burst. The midline defects after maximal gentle approximation ranged from 4 to $10 \mathrm{~cm}$. Patients were followed up for mortality and for early postoperative complications (reburst, sepsis, fistula, abdominal compartment syndrome, skin necrosis). Long-term follow-up was done for development of incisional hernia.

Technique: All patients underwent general anesthesia and received preoperative parenteral antibiotic prophylaxis (cefuroxime 1,500 mg/Clindamycin $600 \mathrm{mg}, 30 \mathrm{~min}$. before surgery). The laparotomy was started at the midline above the laparostomy. A complete adhesiolysis was generally performed to get a clear view on the anatomy and to free the bowel from the abdominal wall. Full exploration of the abdominal cavity was performed. The subsequent abdominal wall closure was performed using the components separation technique as described by Ramirez et al. ${ }^{9}$ The skin and subcutis were separated from the underlying abdominal musculature in lateral direction up to the anterior axillary line. Next, the aponeurosis of the external oblique muscle was incised pararectally. Subsequently, the external oblique muscle was separated from the underlying internal oblique muscle by blunt dissection in a relatively avascular plane. This was followed by the separation of the rectus muscle from the posterior rectus sheath. The separation of the muscle components of the abdominal wall allowed mobilization of each unit over a greater distance with less tension. Subsequently, the abdominal wall was closed using PDS 1 Figures (1-4) with two suction drains routinely used between the mobilized skin and abdominal wall musculature to drain the dead space. The skin was closed because leaving the skin open would not guarantee adequate drainage (via the suction drains) of the large subcutaneous wound surfaces. Therefore, skin closure combined with closed wound drainage was preferred. Postoperatively, no abdominal binders were applied routinely. In case of a wound infection, the running sutures were (partly) removed for adequate drainage.

\section{Results:}

Component separation technique for closure of burst abdomen was done on 20 cases. There were 12 men and 8 women with a mean age of 48.6 years (range, 35-65y) Table (1). One patient died in the fourth postoperative day from pulmonary embolism (mortality 5\%) that was not related to the technique and this patient was excluded from the mean hospital stay and long term follow up. None of the patients needed a ventilator postoperatively. 2 patients of 19 developed wound complication in the form of subincisional abscesses (10.5\%), which were successfully drained. Only 1 patient (5\%) showed skin ischemia with subsequent limited necrosis that was treated by debridement and secondary suture. No single case of reburst occurred. Only the patient with liver cirrhosis and moderate ascites showed few symptoms of abdominal compartment syndrome (tense abdomen and dyspnea) in the early postoperative period, but the patient survived. One patient had a biliary fistula after bile-duct reconstruction for iatrogenic bile duct injury. This was also not related to the technique, and inspite of the wound being continuously soaked with bile from the fistula, causing an overlying skin necrosis, reburst did not occur and the wound healed well, but incisional hernia occurred and was treated later on by surgical repair with synthetic mesh Table (2). The mean hospital stay was 18.5 days, and only the cirrhotic ascitic patient stayed longer (28 days). Two patients were not included in the long-term follow-up analysis (1 patient died and 1 patient was lost to follow-up evaluation). The mean follow-up period for patients ( $\mathrm{n}=18)$ was 12 months (range, $1-24)$.

\section{Discussion:}

There are a number of circumstances on both the trauma and general surgery services when apposition of the fascial edges of the incision is either not feasible or is potentially detrimental. With tissue loss following injury or debridement and abdominal wall retraction, reapproximation of the fascial edges may 


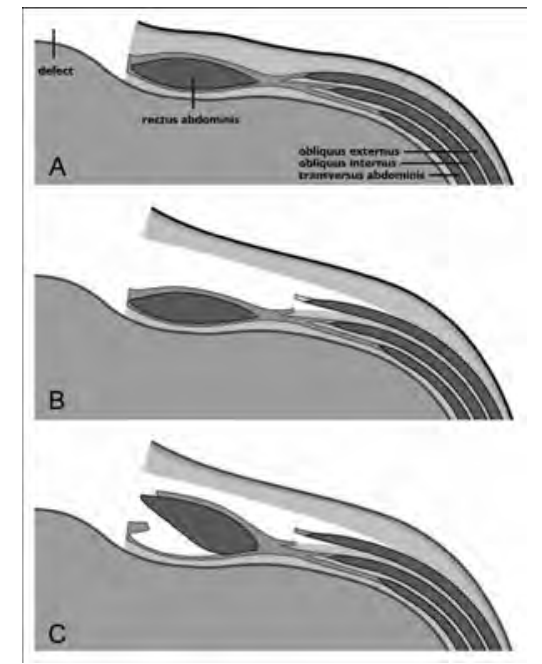

Figure (1): Cross-sectional view. Skin and subcutaneous tissue are separated from the underlying abdominal muscle in lateral direction up to the anterior axillary line. Next the aponeurosis of the external oblique muscle is incised pararectally, and the external oblique muscle is separated from the underlying internal oblique muscle by blunt dissection (A and B). Additionally, (C) the rectus muscle is separated from the posterior rectus sheath.

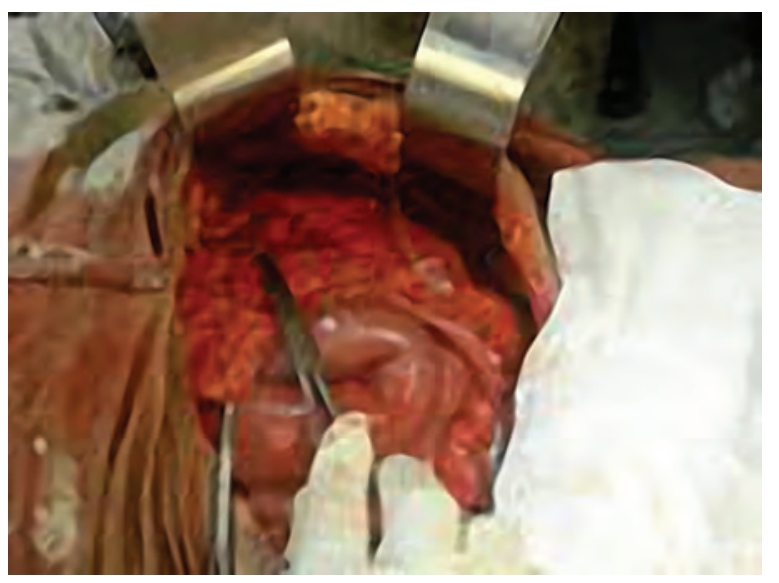

Figure (2): The aponeurosis of the external oblique muscle was incised pararectally.

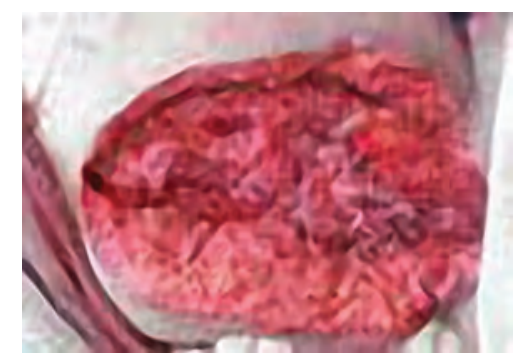

Figure (4): Closure of the defect by mobilized abdominal muscles

be impossible. Similarly, an increase in the volume of intra-abdominal contents due to the insertion of packs or edema of the midgut secondary to resuscitation may preclude

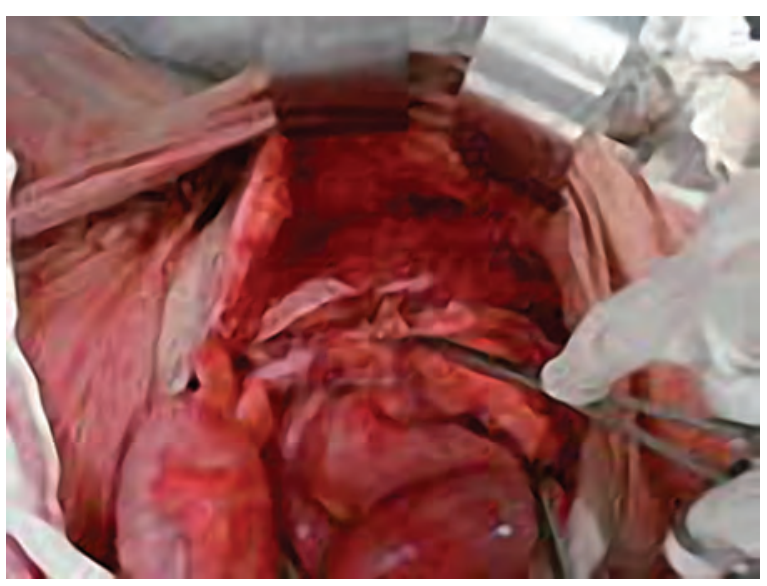

Figure (3): The separation of the rectus muscle from the posterior rectus sheath.

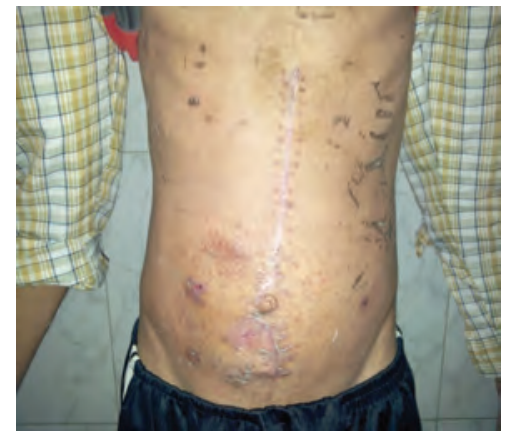

Figure (5): Patient a few months after closure with no hernia.

fascial closure. So any surgeon may, and indeed will be, presented with the inability to close the abdomen. Closing the abdomen under tension may lead to fascial dehiscence, 
Table 1. Original surgery, age, gender, duration of hospital stay and mortality of the study group.

\begin{tabular}{|l|l|l|l|l|l|}
\hline \multicolumn{1}{|c|}{ Original surgery } & Pat. No. & $\mathrm{M} / \mathrm{F}$ & $\begin{array}{c}\text { Age } \\
\text { (mean)/y }\end{array}$ & $\begin{array}{c}\text { Hospital stay } \\
\text { (mean) /day }\end{array}$ & Mortality \\
\hline Anterior resection & 4 & $2 / 2$ & 47 & 15.5 & 0 \\
\hline Left hemicolectomy & 4 & $3 / 1$ & 50 & 17 & 0 \\
\hline Abdominoperineal resection & 3 & $2 / 1$ & 50 & 24 & 0 \\
\hline $\begin{array}{l}\text { Choledochojejunostomy } \\
\text { (iatrogenic CBD injury) }\end{array}$ & 1 & $0 / 1$ & 38 & 21 & 0 \\
\hline Perforated duodenal ulcer & 1 & $1 / 0$ & 65 & 4 & 1 \\
\hline Right hemicolectomy & 4 & $2 / 2$ & 49 & 16 & 0 \\
\hline Traumatic ileal perforation & 2 & $2 / 0$ & 41 & 18 & 0 \\
\hline $\begin{array}{l}\text { Small bowel resection } \\
\text { (volvolus) }^{\mathrm{b}}\end{array}$ & 1 & $0 / 1$ & 53 & 28 & 0 \\
\hline Total & 20 & $12 / 8$ & 48.6 & 18.5 & 1 \\
\hline
\end{tabular}

CBD:common bile duct.

a) This patient died on the 4th postoperative day and was excluded from mean hospital stay and long term follow up.

b) This is the cirrhotic ascitic patient.

Table 2. Incidence, type of complications and mortality.

\begin{tabular}{|l|l|}
\hline Type of complication & No. of patients (\%) \\
\hline Subincisional abscess needing percutaneous drainage & $2(10.5)$ \\
\hline Intra-abdominal collection needing re-exploration & $0(0)$ \\
\hline Gastrointestinal fistula & $0(0)$ \\
\hline Compartment syndrome & $1(5)^{\mathrm{a}}$ \\
\hline Skin necrosis & $1(5)$ \\
\hline Reburst & $0(0)$ \\
\hline Incisional hernia & $1(5.5)$ \\
\hline Mortality & $1(5)^{\mathrm{b}}$ \\
\hline
\end{tabular}

a) Mild symptoms and signs in the patient with cirrhosis.

b) The patient died from pulmonary embolism unrelated to the technique on the 4th postoperative day.

fascial necrosis, skin necrosis, infection of the incision, incisional hernia, and the sequel of the abdominal compartment syndrome. ${ }^{3,11,12}$

To date, no one technique has proven to be appropriate for all circumstances. Because of the high mortality and morbidity rate of burst abdomen, an attempt to prevent it by the use of mass closure had reduced the incidence from $3 \%$ to $1 \% .^{1}$

Many reconstruction methods for abdominal dehiscence have been described, such as direct tissue closure, prosthetic mesh repair, rectus abdominis advanced flap reconstruction, 9,16 tensor fascia lata flap reconstruction, ${ }^{17,18}$ and vacuum-assisted closure. ${ }^{19,20}$

Using a synthetic mesh to bridge the fascial defect usually requires one or more delayed procedure(s) to close the wound or cover the visceral mass with skin graft and results in large abdominal wall hernias. ${ }^{11,21}$ The overall complication rate of polypropylene mesh 
closure approaches $80 \%$, to the extent that some authors recommend covering the mesh with full-thickness skin or muscle flaps in the early postoperative period or even removing the mesh at the earliest time conductive to fascial closure (within 2 weeks) in an attempt to reduce the complication rate. ${ }^{22}$ The use of absorbable mesh shows advantage compared with nonabsorbable mesh, ${ }^{23}$ but incisional hernia is inevitable, and it does not completely prevent development of fistulas. ${ }^{8}$ Myocutaneous flaps or even split-thickness skin coverage of the defect is being attempted by some authors to avoid the late complications of mesh usage. ${ }^{22}$ Bilateral incisions to relax the skin and rectus fascia were proposed. This technique permits medial myocutaneous advancement, up to $10 \mathrm{~cm}$ from each side, and primary tension-free skin closure of midline laparotomy incisions, but it was not clear how this "10 cm" was measured. All patients develop midline wound hernias, with $25 \%$ mortality and 13\% incidence of reburst with very long convalescence (20-180; mean 88 days). The lateral wounds heal with an ugly scar. ${ }^{8}$ The use of a specially designed external tissue expanders to allow delayed primary healing with full thickness skin and subcutaneous tissue. Unfortunately, there is no proper follow-up in this study. ${ }^{24}$ The SAC procedure is a new technique developed by Kafie et al., done only in 3 patients, and needs further evaluation. ${ }^{25}$

Themostimportant aspect of reconstructing a functional abdominal wall is the recreation of the linea alba and achieving midline closure.9,10,13 This allows the abdominal wall to be encompassed by functional muscular components in a manner similar to normal anatomy. As opposed to an inert material, the abdominal musculature provides dynamic support of innervated tissue to redistribute the stress applied from intra-abdominal forces. ${ }^{14}$ The component separation technique not only allows for local tissue transfer but allows for enough midline advancement to close large and complex defects.9,15

As to incisional hernia, whatever the surgical suture technique used to close burst abdomen, the incidence is between 34\% and
$43 \%$ in techniques where the skin and fascia is being closed. ${ }^{26}$ The incidence of ventral hernia from the different techniques that do not involve closure of the fascia is $100 \%{ }^{8}$ Even in planned ventral hernias, the resulting hernia is unsightly huge and difficult to repair. ${ }^{3,6}$ But in our study only one patient (patient of bile duct injury) had incisional hernia $(5.5 \%)$ and was treated later on by surgical repair with synthetic mesh.

None of our patients developed gastrointestinal fistula, but in other studies gastrointestinal fistulas that occurred in relation to abdominal wall closure procedure was about $5 \%^{3}$ and may range from $9 \%-20 \%$ in cases where a prosthetic mesh is used. ${ }^{6}$ One patient had a biliary fistula, which was related to the original problem and primary procedure. Although there was a high output biliary leak into the abdominal wound over the abdominal repair, reburst did not occur, but incisional hernia developed later on.

No single case of reburst occurred in our study in contrast to the study of Tremblay et al., reburst may occur in a significant number of patients (5\%), even in open abdomen techniques, skin only, silo method or mesh application. ${ }^{3}$ Other procedures using myocutaneous advancement and primary skin closure gave a $13 \%$ incidence of reburst. ${ }^{8}$

3 patients out of 19 (16\%) in our study developed wound complications in the form of subincisional abscesses (2 patients) who were drained under general anesthesia and skin necrosis (1 patient) who was treated by debridement and secondary suture. But Karem and Michael in their study show high wound complications rate (52\%) in open group and (27\%) in endoscopic group. ${ }^{28}$ Intra-abdominal abscess is an unavoidable complication, even in temporary abdominal closure techniques $(4.5 \%)^{27}$, but in our study there was no case of intra-abdominal abscess. However in the study of Levy et al., recurrent intra-abdominal abscess formation was around $13 \%$; a minority usually drained spontaneously, and most patients required reoperation, with a mortality rate of $55 \%{ }^{8}$

Only one patient died in the fourth postoperative day from pulmonary embolism 
(mortality 5\%) that was not related to the technique. The problem is that mortality varies with the underlying disease. According to the available literature, mortality of patients with parenchymatous liver disease who develop burst abdomen, although few, was $100 \% .^{3}$ Mortality in such patients is due to abdominal compartment syndrome, reburst with ascitic leak, wound infection, and hepatorenal failure. Other references did not mention the survival rate of patients with hepatic insufficiency among their groups of burst abdomen, nor did they mention the degree of insufficiency (whether the patients were ascitic or not). ${ }^{8}$

It should be noted that the vast majority of published studies are retrospective, discuss a single technique and include fewer than 15 patients a year. As no prospective randomized trials exist, it remains unclear, given the small number of patients in the various studies, what the rate of mortality, incidence of complications and rate of fascial closure are with the various techniques. ${ }^{3}$

Concerning cost, our method is a simple, one-stage procedure without prosthesis or multistage surgery. Also, the hospital stay usually does not exceed 3 weeks unless there is an associated clinical problem. None of the patients needed re-exploration for intraabdominal sepsis and subsequently low cost.

\section{Conclusion:}

As the ideal technique for closure of burst abdomen should be technically so simple that the results are as good in the hands of the trainee as in those of the surgical master, it should be free from complications, comfortable to the patient and leave a reasonable aesthetic scar. These criteria fit with the component separation technique in which the peritoneal integrity and defenses are maintained, postoperative care is simplified with comparable mortality and morbidity for closure of burst abdomen.

\section{Reference:}

1- Bucknall TE, Cox PJ, Harold E: Burst abdomen and incisional hernia: A prospective study of 1129 major laparotomies. Br Med J
(Clin Res Ed) 1982; 284(6320): 931-933.

2- Madsen G, Fischer L, et al: Burst abdomenclinical features and factors influencing mortality. Dan Med Bull 1992; 39(2): 183-185(Abs).

3- Tremblay LN, Feliciano DV, Schmidt J, et al: Skin only or silo closure in the critically ill patient with an open abdomen. Am J Surg 2001; 182(6): 670-675.

4- Haddad V, Macan WL IV: Abdominal wound dehiscence and evisceration: Contributing factors and improved mortality. Am Surg 1980; 46(9): 508-513.

5- Brock WB, Barker DE, Burns RP: Temporary closure of open abdominal wounds: The vacuum packs. Am Surg 1995; 61(1): 30-35.

6- Fabian TC, Corce MA, Pritchard FE, et al: Planned ventral hernia staged management for acute abdominal wall defects. Ann Surg June 1994; 219(6): 643-653.

7- Luis O, Jae-Wook OH: Flaps and other reconstructive techniques. In: Mastery of surgery, Nyhus LM, Baker RJ, Fischer JE, (Editors); Boston, Little, Brown\& Company (Publishers); $3^{\text {rd }}$ edn. vol. I, p. 288.

8- Levy E, Palmer DL, Frileux P, et al: Septic necrosis of the midline wound in postoperative peritonitis. Successful management by debridement, myocutaneous advancement and primary skin closure. Ann Surg 1988; 207(4): 470-479.

9- Ramirez OM, Ruas E, Dellon AL: "Components separation" method for closure of abdominal-wall defects: An anatomic and clinical study. Plast Reconstr Surg 1990; 86: 519-526.

10- Shestak KC, Edington HJ, Johnson RR: The separation of anatomic components technique for the reconstruction of massive midline abdominal wall defects: Anatomy, surgical technique, applications, and limitations revisited. Plast Reconstr Surg 2000; 105: 731-738.

11- Smith PC, Tweddell JS, Bessey PQ: Alternative approaches to abdominal wound closure in severely injured patients with massive visceral edema. J Trauma 1992; 32(1):16-20.

12- Ertel W, Oberholzer A, Platz A, et al: Incidence and clinical pattern of the abdominal compartment syndrome after "damage-control” laparotomy in 311 patients with severe abdominal and/or pelvic trauma. Crit Care Med 2000; 28(6): 1747-1753.

13- Nguyen V, Shestak KC: Separation of 
anatomic components method of abdominal wall reconstruction-clinical outcome analysis and an update of surgical modifications using the technique. Clin Plast Surg 2006; 33: 247-257.

14- Sukkar SM, Dumanian GA, Szczerba SM, et al: Challenging abdominal wall defects. Am J Surg 2001; 181: 115-121.

15- Lowe JB, Garza JR, Bowman JL, et al: Endoscopically assisted "components separation" for closure of abdominal wall defects. Plast Reconstr Surg 2000; 105: 720-730.

16- DeFranzo AJ, Kingman GJ, Sterchi JM, Marks MW, Thorne MT: Rectus turnover flaps for the reconstruction of large midline abdominal wall defects. Ann Plast Surg 1996; 37: 18-23.

17- Nahai F, Silverton JS, Hill HL, Vasconez LO: The tensor fascia lata musculocutaneous flap. Ann Plast Surg 1978; 1: 372-379.

18- Hayami S, Hotta T, Takifuji K, Iwahashi M, Mitani Y, Yamaue H: Reconstruction of an infected recurrent ventral hernia after a mesh repair using a pedicled tensor fascia lata flap: Report of two cases. Surg Today 2009; 39: 811-817.

19- Miller PR, Meredith JW, Johnson JC, Chang MC: Prospective evaluation of vacuumassisted fascial closure after open abdomen: Planned ventral hernia rate is substantially reduced. Ann Surg 2004; 239: 608-614.

20- Heller L, Levin SL, Butler CE: Management of abdominal wound dehiscence using vacuum assisted closure in patients with compromised healing. Am J Surg 2006; 191:
165-172.

21- Jones JW, Jurkovich GJ: Polypropylene mesh closure of infected abdominal wounds. Am Surg 1989; 55(1):73-76.

22- Stone HH, et al: Management of acute fullthickness losses of the abdominal wall. Ann Surg 1981; 193: 612-618.

23- Gross E, Erhard J, Eigler FW: Artificial mesh as an aid in abdominal wall closure in postoperative peritonitis, postoperative abdominal wall dehiscence and reconstruction of the abdominal wall. Zentralbl Chir 1984; 109(19): 1238-1250 (Article in German).

24- Schessel ES, et al: The management of the postoperative disrupted abdominal wall. Am J Surg 2002; 184(3): 263-268.

25- Kafie FE, Tessier DJ, Williams RA, et al: Serial abdominal closure technique (the "SAC" procedure): A novel method for delayed closure of the abdominal wall. Am Surg 2003; 69(2):102-105.

26- Gislason H, VisteA: Closure of burst abdomen after major gastrointestinal operationscomparison of different surgical techniques and later development of incisional hernia. Eur J Surg 1999; 165(10): 958-961.

27- Barker Donald E, Kaufman Henry J, Smith Lisa A, et al: Vacuum Pack technique of temporary abdominal closure: A 7 year experience with 112 patients. J Trauma 2002; 48(2): 201-207.

28- Harth KC, Rosen MJ: Endoscopic versus open component separation in complex abdominal wall reconstruction. Am J Surg 2010; 199: 342-347. 